\title{
Measurement of Bubble Behavior and Impact on Solid Wall Induced by Fiber-Holmium:YAG Laser
}

\author{
Yasuhiro Sugimoto', Yoshiaki Yamanishi', Keiichi Sato', Manabu Moriyama² \\ ${ }^{1}$ Department of Mechanical Engineering, Kanazawa Institute of Technology, Ishikawa, Japan \\ ${ }^{2}$ Himi Municipal Hospital, Kanazawa Medical University, Toyama, Japan \\ Email: y-sugi@neptune.kanazawa-it.ac.jp
}

Received 26 April 2015; accepted 28 August 2015; published 7 October 2015

Copyright (C) 2015 by authors and Scientific Research Publishing Inc.

This work is licensed under the Creative Commons Attribution International License (CC BY). http://creativecommons.org/licenses/by/4.0/

c) (i) Open Access

\begin{abstract}
The holmium:YAG (Ho:YAG) laser is effectively used for transurethral ureterolithotripsy. The laser is applied through an optical fiber in a ureter. A bubble is formed by a laser irradiated from the fiber tip and a calculus is crushed by the impact of the bubble collapse. In this study, we observed the characteristic behavior of a bubble induced by a Ho:YAG laser near a wall surface, using a high-speed video camera. Furthermore, we measured the forces of a bubble collapse using an impulsive force sensor. As a result, we showed characteristic bubble collapse behavior and impulsive force distribution for various fiber placement conditions.
\end{abstract}

\section{Keywords}

Laser-Induced Cavitation Bubble, Bubble Collapse, Shock Waves, Transurethral Ureterolithotripsy

\section{Introduction}

For urinary stone disease treatment using shock waves, there are several effective methods of lithotripsy such as extracorporeal shock wave lithotripsy (ESWL) [1], laser lithotripsy [2]-[7], and electrohydraulic lithotripsy (EHL) [7]. We focus on bubble behavior induced by a fiber laser used for transurethral ureterolithotripsy (TUL) [2] [6], and its resulting impact on a solid wall surface. In TUL, a single bubble is formed by a laser irradiated from the tip of a fiber, and shock waves formed by the collapse of the bubble propagate toward the stone.

There are many studies on the characteristic behavior and impact of laser-induced cavitation bubble collapse near a solid wall. There are also detailed observations for a single bubble induced by a focused laser that show characteristic collapse behavior and impact [8]-[13]. Those results show the existence of interesting impulsive 
behavior (such as a shock wave and a micro jet) associated with the mechanism of the impact and the erosion caused by a collapse of single bubble. In addition, behaviors of cavitation bubble collapse in flow field are also shown by using a high speed video camera synchronized with the impulsive force sensor and hydrophones [14]-[16]. Recently, there are many numerical studies on a single bubble collapse with associate to mechanism of impact [12] [17]-[20]. In the viewpoint of medical field, the effects of a nearby elastic wall surface on bubble collapse behavior have been made clear experimentally and numerically [17] [18] [21]. These show the characteristic bubble collapse with shock wave emission and micro-jet penetration.

In this study, we conduct several experiments to examine bubble behavior and its impact on a nearby solid wall following fiber laser-induced bubble collapse. Although there are some studies on the behavior of bubbles induced by a fiber laser [2] [4], there appears to be few studies for in vivo treatment with detailed high-speed observations. In this study, we investigate the effects of laser irradiation conditions (laser power and fiber diameter) with and without solid boundaries (distance between the wall and fiber tip) to reveal the optimum laser irradiation conditions for the treatment of urinary stone disease.

\section{Experimental Apparatus and Procedure}

Figure 1 shows the experimental setup. The holmium:YAG (Ho:YAG) laser used in this experiment is applied in a vessel (100 $\mathrm{mm}$ in height, $120 \mathrm{~mm}$ in width and $50 \mathrm{~mm}$ in depth) filled with tap water (temperature $T_{w}$, dissolved oxygen content $\beta$ ) through a fiber (fiber diameters $d=360,420$, and $550 \mu \mathrm{m}$ ) connected to a laser source (Lumenis, VersaPulse Holmium YAG LASER 30W). The wave length and pulse duration of the laser are $\lambda=$ $2.06 \mu \mathrm{m}$ and $\tau=250 \mu \mathrm{s}$, respectively. The energy of the laser is set to $E=0.5,1.0,1.5$, and $2.0 \mathrm{~J}$ per pulse. The energy is immediately absorbed in water as heat due to the relatively long wavelength, and then a bubble is formed [4]. A laser-induced bubble is illuminated by a metal-halide light and observed by a high-speed video camera (Photron, SA5) located on the opposite side of the light. The frame speed of the camera is Fs = 100,000 frames per second (fps). The camera is synchronized with a hydrophone (B \& K, 8013) that has flat frequency response within $\pm 3 \mathrm{~dB}$ over the frequency range from $0.1 \mathrm{~Hz}$ to $180 \mathrm{kHz}$ or a hand-made impulsive force sensor and can record the bubble aspect before and after the laser irradiation [14]-[16].

Figure 2 shows a schematic diagram of a handmade PVDF (Polyvinylidene difluoride) impulsive force sensor. The PVDF film is fixed by an adhesive on an acrylic resin plate. The sensing area is made of an epoxy resin with $4 \mathrm{~mm}$ in diameter. The sensing area is covered by a silicone tube and a silicone gasket so as not to respond to impacts on the surrounding area. The fiber tip is placed on the center axis of the impulsive force sensor. The diameter of the sensor is sufficiently large relative to the bubble size at the collapse. Figure 3 shows output waveforms of the handmade sensor. Figure 3(a) is results in ball drop test (the ball is made of glass, mass $m=$ $4.6 \mathrm{~g})$ ), and Figure 3(b) is in cavitation bubble. Both pulses rise just after the impact and then swing to the negative value due to the sensor characteristics. The handmade sensor is suitable for cavitation impulse measurements since the wave form rise is within about $5 \mu$ s as shown in Figure 3(b). Here, since the pulse duration is important for the calibration [22], the calibration must be improved further.

The laser fiber is installed perpendicular (vertical wall surface) or parallel (horizontal wall surface) to the solid

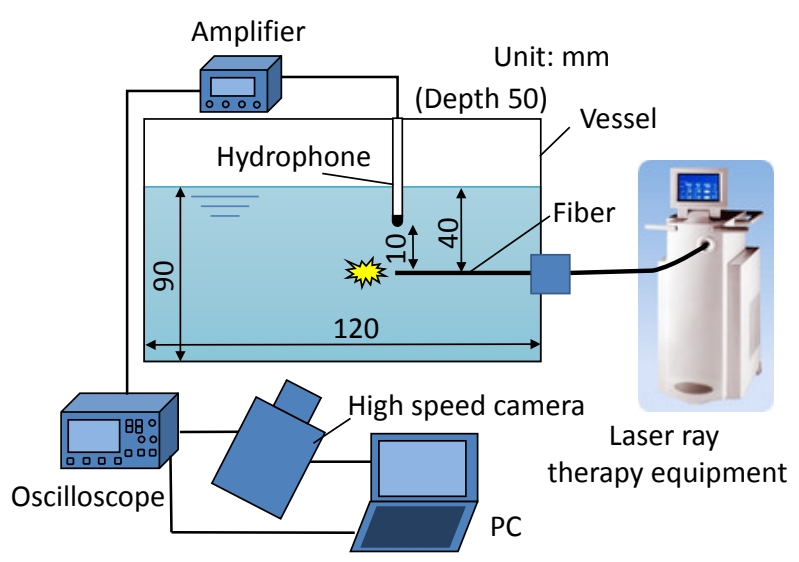

Figure 1. Experimental setup. 


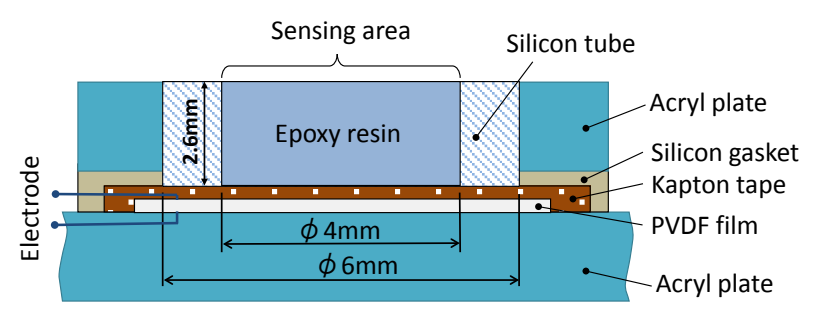

Figure 2. Schematic diagram of impulsive force sensor.

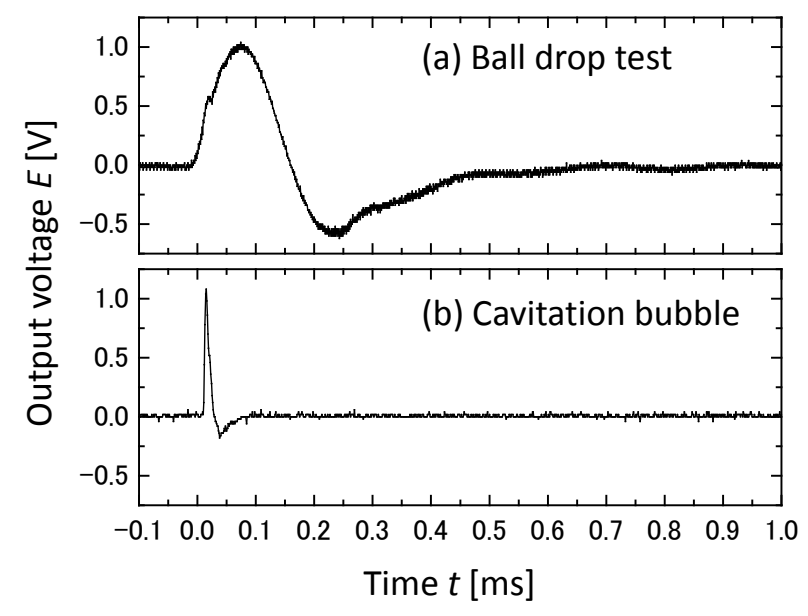

Figure 3. Output of impulsive force sensor.

wall with the impulsive force sensor, as shown in Figure 4. The distance between the fiber tip and the wall surface is set as $x$ for the former case or $h=0$ to $5.5 \mathrm{~mm}$ for the latter case.

\section{Results}

\subsection{Bubble Behavior and Impact in the Absence of a Wall Surface}

Figure 5 shows the behavior of bubble formation and collapse in the absence of a wall surface. For all laser irradiation conditions, a single bubble forms immediately after irradiation $(t=0.05 \mathrm{~ms})$ before reaching its maximum diameter and then collapsing with multiple rebounds. Figure 6 shows a time course of equivalent bubble radius with various fiber diameter and laser energy conditions. The bubble radius is equivalent radius calculated from the projected area in each image assuming axial symmetry. The maximum bubble radius $R_{\max }$ becomes larger with an increase in laser power and fiber diameter. The life of the bubble also becomes longer with the increase in the maximum bubble radius. The bubble collapses and then rebounds with some radius because a bubble contains vapor and non-condensable gas. Here Rayleigh's bubble collapse time $t_{c}$, i.e., the time from the bubble maximum to the subsequent minimum, can be given by Equation (1) [11],

$$
t_{c}=0.915 R_{\max } \sqrt{\rho /\left(P_{0}-P_{v}\right)}
$$

where $\rho$ is the density of water, $P_{0}$ is ambient pressure of the bubble, and $P_{v}$ is the saturated pressure of water.

Figure 7 shows bubble collapse times calculated using Figure 6 and Equation (1). There is a tendency for these bubble collapse times to be longer than the values given by Equation (1) due to containing non-condensable gas. Figure 8 shows changes in sound pressure measured by the hydrophone installed at $10 \mathrm{~mm}$ from the fiber tip. The sound pressure becomes larger with an increase in the maximum bubble radius. This tendency also agrees with previous studies [3]. The bubble becomes larger with an increase in laser power as shown in Figure 6, namely, the energy is stored in the bubble. The bubble will be compressed back to a small volume inverting the expansion. The energy is lost as a shock wave that is launched into the liquid upon collapse of the bubble. Since these studies have been carried out in many past studies, we transfer more detailed mechanism to reference [9]. 


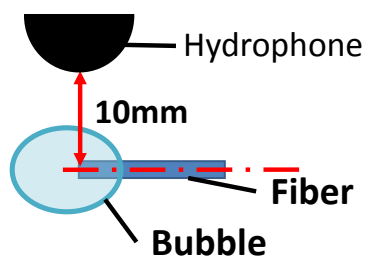

(a) Without wall

Wall (Sensor)

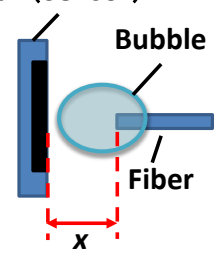

(b) Vertical wall surface

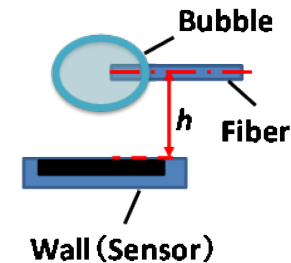

(c) Horizontal wall surface

Figure 4. Arrangement of laser fiber and impulsive force sensor.

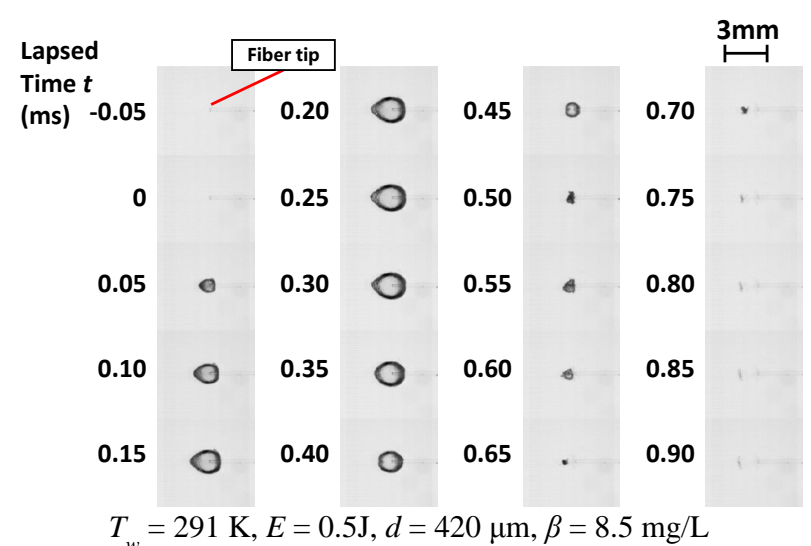

Figure 5. Bubble formation and collapse behavior.

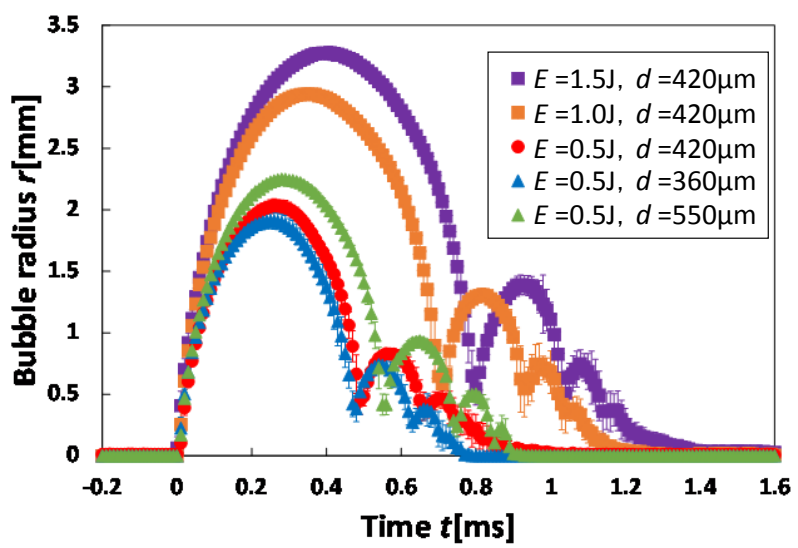

$$
T_{W}=292 \mathrm{~K}, \beta=7.2 \mathrm{mg} / \mathrm{L}, F s=100000 \mathrm{fps}
$$

Figure 6. Time course of bubble radius.

\subsection{Bubble Behavior and Impact near a Wall Surface}

Figure 9 shows examples of bubble collapse behavior and the associated impact waves near the solid wall that 


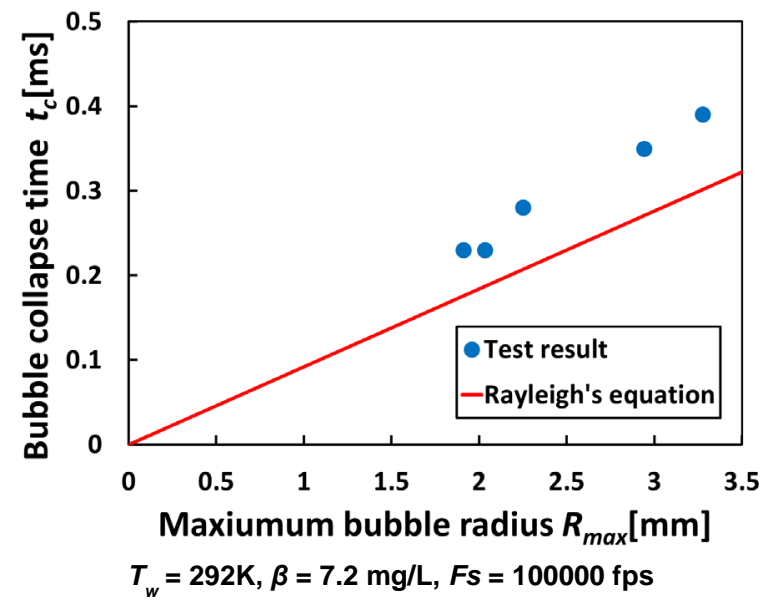

Figure 7. Changes in bubble collapse time.

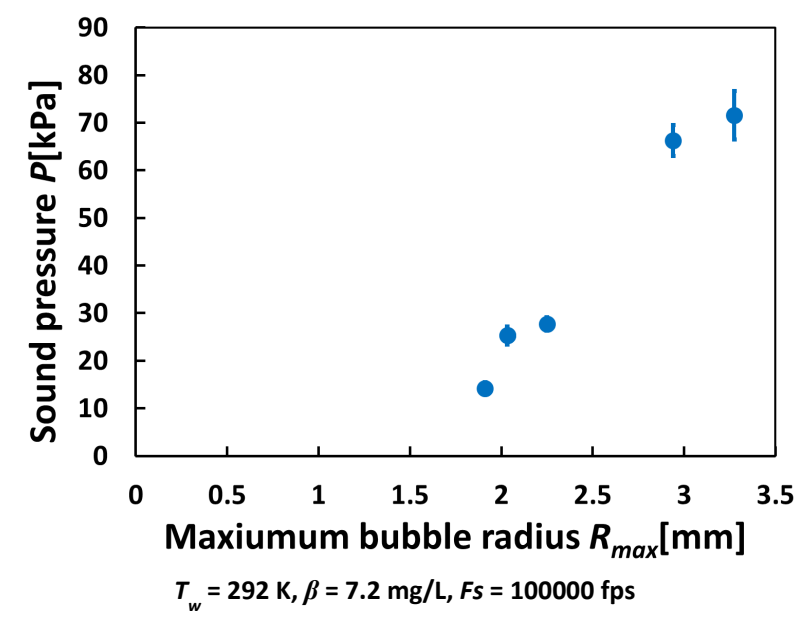

Figure 8. Changes in sound pressure against maximum bubble radius.

is perpendicular to the laser fiber. The bubble is formed in contact with the wall and collapses on the wall surface when the laser fiber is placed close to the wall as shown in Figure 9. Even when the fiber tip is placed at a distance from the wall, the bubble moves toward the wall with a collapsing and rebounding motion as shown in Figure 9(b) and Figure 9(c). The lower parts of Figures 9(a)-(c) show the resultant impulsive force measured by the impulsive force sensor synchronized with high-speed observation. The impulsive force reaches a peak value at the primary collapse of the bubble rather than at the instant of laser irradiation. Figure 10 shows bubble collapse behavior and the associated impact waves near the wall that is parallel to the fiber. The non-spherical bubble forms in contact with the wall surface and collapses toward the attaching wall surface when the laser fiber is placed close to the wall, as shown in Figure 10(a). The impulsive force also reaches a peak value at the point of primary collapse of the bubble in each condition.

For the vertical and horizontal wall surfaces, respectively, Figure 11 and Figure 12 show the relationship between impulsive force $F$ and distance $\gamma\left(=x / R_{\max }\right.$ or $\left.h / R_{\max }\right)$ from the fiber tip to the wall surface. These plots are the average of 10 times and the error bars indicates the standard deviation. The impulsive force becomes larger with a decrease in distance $\gamma$. The magnitude of this impact is comparable with previous studies (such as reference [11]) and can cause erosion on the material surface. In the case of the fiber arranged perpendicular to the wall, the impulsive force reaches its maximum value at a certain distance because placing the laser fiber directly at the wall limits cavitation bubble expansion, thereby restricting the amplitude of the resultant shock wave. Therefore, it is found that the high impact on the wall can be given effectively by the optimum distance 


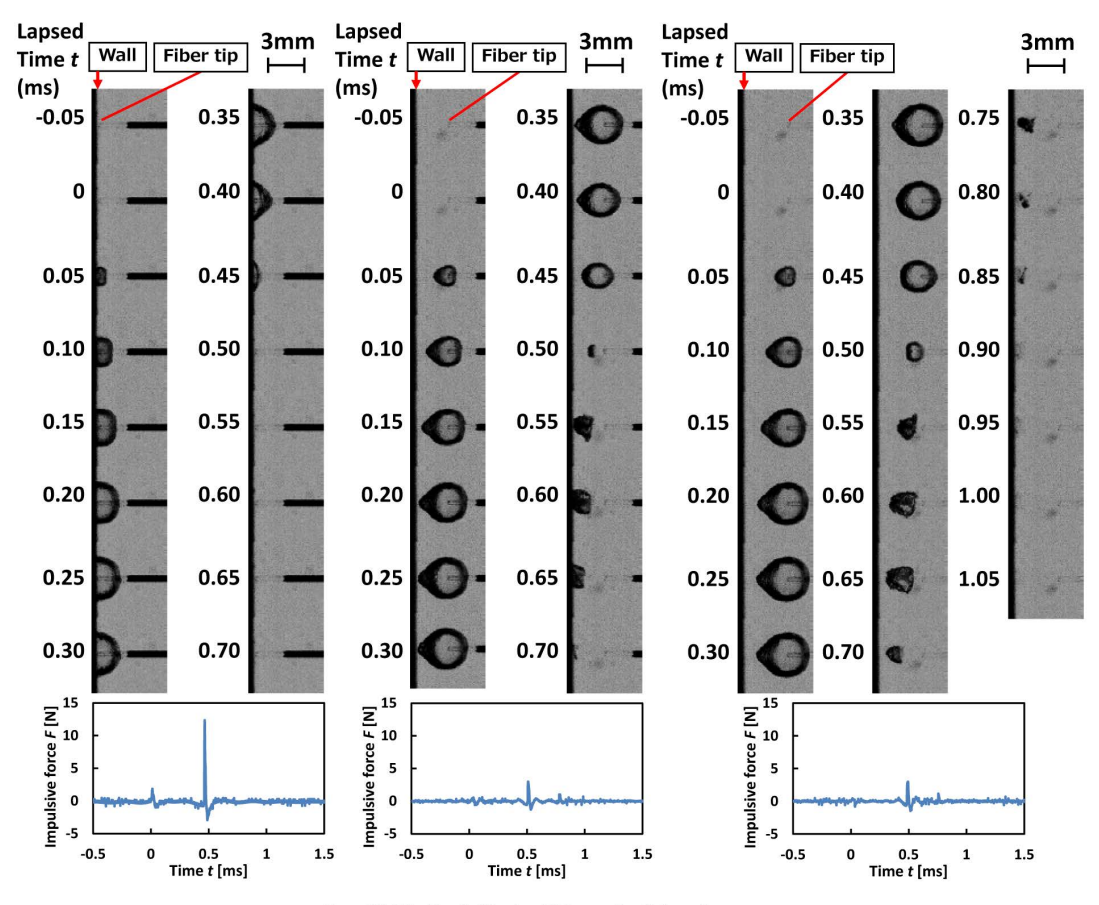

(a)

(b)

(c)

Figure 9. Bubble collapse behavior and the resultant impulsive force near a vertical wall surface. (a) $x=0.33 \mathrm{~mm}$; (b) $x=2.83 \mathrm{~mm}$; (c) $x=3.79 \mathrm{~mm}$.

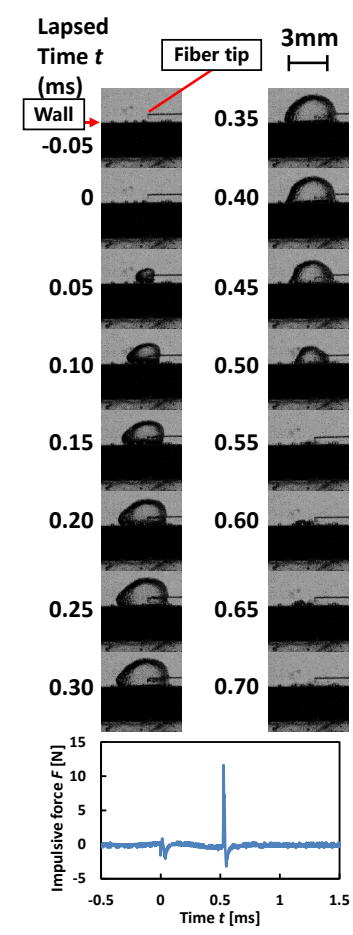

(a)

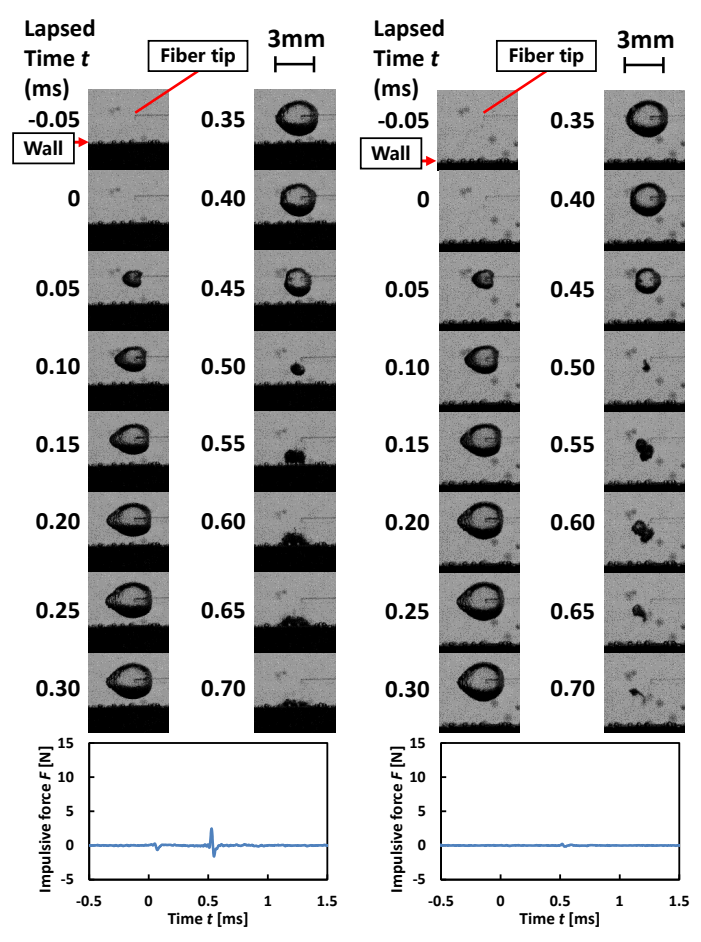

$T_{w}=292 \mathrm{~K}, \quad E=0.5 \mathrm{~J}, \quad d=420 \mu \mathrm{m}, \quad \beta=8.2 \mathrm{mg} / \mathrm{L}$

(b)

(c)

Figure 10. Bubble collapse behavior and the resultant impulsive force near a horizontal wall surface. (a) $h=0.28 \mathrm{~mm}$; (b) $h=3.11 \mathrm{~mm}$; (c) $h=4.03 \mathrm{~mm}$. 


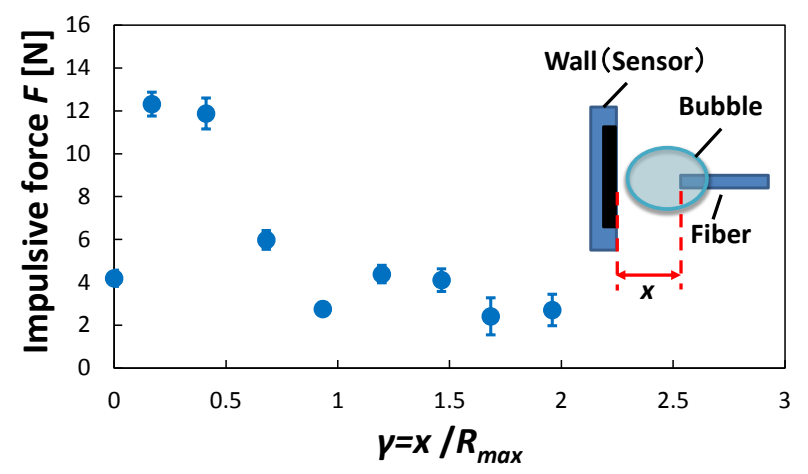

$T_{w}=291 \mathrm{~K}, E=0.5 \mathrm{~J}, d=420 \mu \mathrm{m}, \beta=8.5 \mathrm{mg} / \mathrm{L}$

Figure 11. Changes in impulsive force (vertical wall surface).

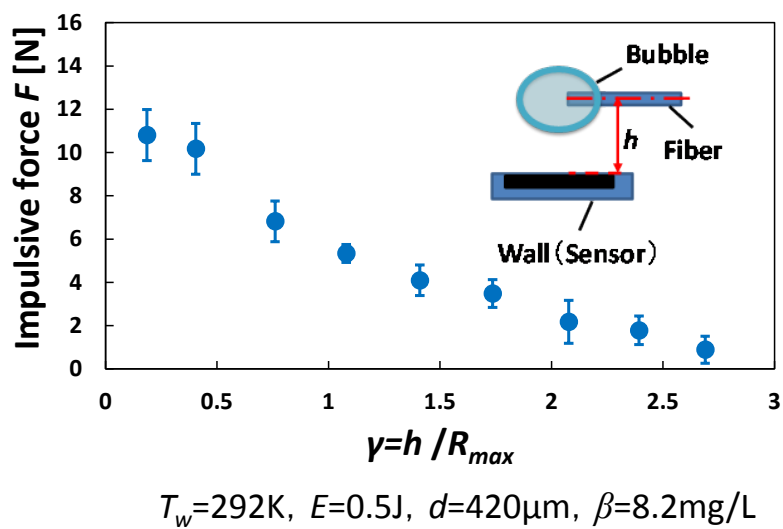

Figure 12. Changes in impulsive force (horizontal wall surface).

from a wall surface.

\section{Conclusions}

The bubble induced by a Ho:YAG fiber laser for a transurethral ureterolithotripsy is observed in detail using a high-speed video camera. Characteristic collapsing behavior of the bubble near the wall and its resulting impulsive force on the wall are found for various laser irradiation conditions.

1) The maximum bubble radius becomes larger with an increase in laser power and fiber diameter.

2) The impulsive force reaches its maximum value at the point of primary collapse of the bubble rather than at the instant of laser irradiation.

3) In the case of the fiber arranged perpendicular to the wall, there is the optimum fiber placement to give high impact on the wall surface because the cavitation bubble expansion and the resultant shock wave are limited by the existence of the wall.

\section{Acknowledgements}

This work was supported by JSPS KAKENHI Grant Number 24560211.

\section{References}

[1] Yoshizawa, S., Ikeda, T., Ito, A., Ota, R., Takagi, S. and Matsumoto, Y. (2009) High Intensity Focused Ultrasound Lithotripsy with Cavitating Microbubbles. Medical \& Biological Engineering \& Computing, 47, 851-860.

http://dx.doi.org/10.1007/s11517-009-0471-y 
[2] Frenz, M., Könz, F., Pratisto, H., Weber, H.P., Silenok, A.S. and Konov, V.I. (1998) Starting Mechanisms and Dynamics of Bubble Formation Induced by a Ho Yttrium Aluminum Garnet Laser in Water. Journal of Applied Physics, 84, 5905-5913. http://dx.doi.org/10.1063/1.368906

[3] Zhong, P., Tong, H.-L., Cocks, F.H. and Preminger, G.M. (1998) Transient Cavitation and Acoustic Emission Produced by Different Laser Lithotripters. Journal of Endourology, 12, 371-378. http://dx.doi.org/10.1089/end.1998.12.371

[4] Vassar, G.J., et al. (1999) Holmium YAG Lithotripsy Photothermal Mechanism. Journal of Endourology, 13, $181-190$. http://dx.doi.org/10.1089/end.1999.13.181

[5] Jamaluddin, A.R., Ball, G.J., Turangan, C.K. and Leighton, T.G. (2011) The Collapse of Single Bubbles and Approximation of the Far-Field Acoustic Emissions for Cavitation Induced by Shock Wave Lithotripsy. Journal of Fluid Mechanics, 677, 305-341. http://dx.doi.org/10.1017/jfm.2011.85

[6] Marks, A.J., Qiu, J., Milner, T.E., Chan, K.F. and Teichman, J.M.H. (2011) Urinary Tract Stone Disease. Springer, Berlin, 301-309.

[7] Delale, C.F. (2013) Bubble Dynamics \& Shock Waves. Springer, Berlin, 291-338. http://dx.doi.org/10.1007/978-3-642-34297-4

[8] Lauterborn, W. and Bolle, H. (1975) Experimental Investigations of Cavitation-Bubble Collapse in the Neighborhood of a Solid Boundary. Journal of Fluid Mechanics, 72-2, 391-399. http://dx.doi.org/10.1017/S0022112075003448

[9] Vogel, A., Lauterborn, W. and Timm, R. (1989) Optical and Acoustic Investigation of the Dynamics of Laser-Produced Cavitation Bubbles near a Solid Boundary. Journal of Fluid Mechanics, 206, 299-338. http://dx.doi.org/10.1017/S0022112089002314

[10] Tomita, Y. and Shima, A. (1986) Mechanisms of Impulsive Pressure Generation and Damage Pit Formation by Bubble Collapse. Journal of Fluid Mechanics, 169, 535-564. http://dx.doi.org/10.1017/S0022112086000745

[11] Delale, C.F. (2013) Bubble Dynamics \& Shock Waves. Springer, Berlin, 67-103.

[12] Lind, S.J. and Phillips T.N. (2012) The Influence of Viscoelasticity on the Collapse of Cavitation Bubbles near a Rigid Boundary. Theoretical and Computational Fluid Dynamics, 26, 245-277. http://dx.doi.org/10.1007/s00162-011-0227-9

[13] Geers, T.L., Lagumbay, R.S. and Vasilyev, O.V. (2012) Acoustic-Wave Effects in Violent Bubble Collapse. Journal of Applied Physics, 112, Article ID: 054910. http://dx.doi.org/10.1063/1.4748870

[14] Sato, K. and Ogawa, N. (1995) Collapsing Behavior of Vortex Cavitation Bubbles in the Wake of a Circular Cylinder. ASME FED (American Society of Mechanical Engineers, Fluids Engineering Division), 226, 119-125.

[15] Sato, K. and Kondo, S. (1996) Collapsing Behavior of a Vortex Cavitation Bubble near Solid Wall: Spanwise-View Study. ASME FED (American Society of Mechanical Engineers, Fluids Engineering Division), 236, 485-490.

[16] Sato, K., Sugimoto, Y. and Ohjimi, S. (2009) Pressure-Wave Formation and Collapses of Cavitation Clouds Impinging on Solid Wall in a Submerged Water Jet. Proceedings of the 7th International Symposium on Cavitation, Ann Arbor, 17-22 August 2009, 1074-1085.

[17] Brujan, E.-A., Nahen, K., Schmint, P. and Vogel, A. (2001) Dynamics of Laser-Induced Cavitation Bubbles near an Elastic Boundary. Journal of Fluid Mechanics, 433, 251-281. http://dx.doi.org/10.1017/S0022112000003347

[18] Brujan, E.-A., Nahen, K., Schmint, P. and Vogel, A. (2001) Dynamics of Laser-Induced Cavitation Bubbles near Elastic Boundaries: Influence of the Elastic Modulus. Journal of Fluid Mechanics, 433, 283-314. http://dx.doi.org/10.1017/S0022112000003335

[19] Kobayashi, K., Kodama, T. and Takahira, H. (2011) Shock Wave-Bubble Interaction near Soft and Rigid Boundaries during Lithotripsy: Numerical Analysis by the Improved Ghost Fluid Method. Physics in Medicine and Biology, 56, 6421-6440. http://dx.doi.org/10.1088/0031-9155/56/19/016

[20] Fortes-Patella, R., Challier, G., Reboud, J.L. and Archer, A. (2013) Energy Balance in Cavitation Erosion: From Bubble Collapse to Indentation of Material Surface. Journal of Fluids Engineering, 135, Article ID: 011303. http://dx.doi.org/10.1115/1.4023076

[21] Kodama, T. and Tomita, Y. (2000) Cavitation Bubble Behavior and Bubble-Shock Wave Interaction near a Gelatin Surface as a Study of in Vivo Bubble Dynamics. Applied Physics B, 70, 139-149. http://dx.doi.org/10.1007/s003400050022

[22] Soyama, H., Lichtarowicz, A., Momma, T. and Williams, E.J. (1998) A New Calibration Method for Dynamically Loaded Transducers and Its Application to Cavitation Impact Measurement. Journal of Fluids Engineering, 120, 712718. http://dx.doi.org/10.1115/1.2820728 


\section{Nomenclature}

$d$ : Fiber diameter $(\mathrm{mm})$

$E$ : Laser power (J)

$F$ : Impulsive force $(\mathrm{N})$

$h$ : Distance from fiber tip to surface of horizontal wall (mm)

$r$ : Equivalent bubble radius (mm)

$R_{\text {max }}$ : Maximum bubble radius (mm)

$t_{c}$ : Rayleigh's bubble collapse time in Ref. [11] (s)

$T_{w}$ : Temperature of water (K)

$x$ : Distance from fiber tip to surface of vertical wall (mm)

$\beta$ : Dissolved oxygen content (mg/L)

$\gamma$ : Ratio of distance from fiber tip to wall surface to maximum bubble radius $\left(=x / R_{\max }\right.$ or $\left.h / R_{\max }\right)$ 